\title{
Decentralized Diagnosis of Networked Discrete Event Systems Subject to Denial of Service Attacks *
}

\author{
Marcos V. S. Alves* Raphael J. Barcelos* \\ Lilian K. Carvalho* João C. Basilio* \\ * Universidade Federal do Rio de Janeiro, Electrical Engineering \\ Program, 21949-900, Rio de Janeiro, Brazil \\ (e-mails: mvalves@poli.ufrj.br; raphajulio@gmail.com; \\ lilian@dee.ufrj.br; basilio@dee.ufrj.br)
}

\begin{abstract}
In this paper, we address the problem of decentralized failure diagnosis of networked discrete event systems (NDES) in the presence of Denial-of-Service (DoS) attacks. We propose an automaton model for NDES subject to DoS attacks that represent the adverse effects of DoS attacks on the observations of local diagnosers. We formulate a new diagnosis property, to be referred here to as DoS-robust codiagnosability, and present a systematic way to verify whether the language generate by a NDES is DoS-robustly codiagnosable.
\end{abstract}

Keywords: Discrete event systems, automata, failure diagnosis, denial-of-service attacks.

\section{INTRODUCTION}

The growth in the use of devices connected through communication networks, for monitoring and control of cyber-physical systems (CPS), has increased the vulnerability of these systems to the action of malicious agents (Pasqualetti et al., 2013). Such systems, when represented by discrete event models, are referred, in the literature, to as Networked Discrete Event Systems (NDES) (Lin, 2014; Alves et al., 2017; Viana et al., 2017; Nunes et al., 2018).

NDES subject to network attacks have been studied by Góes et al. (2017), Carvalho et al. (2018), Su (2018) and Lima et al. (2019). In those works, it is assumed that the malicious agents can insert and/or remove packets with event occurrence information, but they cannot delay the transmission of legitimate packets sent through the communication network. In this paper, we consider Denial-of-Service (DoS) attacks (Kurose and Ross, 2013; Zargar et al., 2013; Lohachab and Karambir, 2018), where malicious agents are able to flood some communication channels with packets, so as to disrupt (temporally or definitely) the services provided by them, and thus, besides they cause effects similar to insertion and removal of event occurrences, they can also cause observation delays. To the best of the authors knowledge, there is no previous work that considers DoS attacks in the context of NDES.

In this paper, we address the problem of decentralized failure diagnosis (Sampath et al., 1995; Debouk et al., 2000), where we want to be sure that we can detect an occurrence of a failure event, within a bounded number

\footnotetext{
^ This work has been supported in part by the Coordenação de Aperfeiçoamento de Pessoal de Nível Superior (CAPES), Finance Code 001, and the Brazilian Research Council (CNPq), grant number $309.652 / 2017-0$.
}

of event occurrences, in the presence of DoS attacks on vulnerable communication channels used to transmit event occurrences from the plant to the diagnosers. We assume that the malicious agents apply a data flooding strategy (Zargar et al., 2013), by inserting both, meaningless packets and valid packets with false information, into the vulnerable channels. As a consequence, the DoS attacks can: (i) lead a diagnoser to observe event occurrences lately, (ii) create false event observations, and, (iii) cause loss of observations by overflowing channel buffers.

The structure of the paper is as follows. In Section 2 we present some preliminary concepts. In Section 3 we introduce the structure of NDES subject to DoS attacks. In Section 4 we propose an automaton model for NDES subject to DoS attacks. In Section 5, we first propose a new diagnosis property that takes into account DoS attacks, and, in the sequel, we present an algorithm for its verification. The conclusions are drawn in Section 6 .

\section{BACKGROUND PRELIMINARIES}

The formalism used here to model DES is the so-called (deterministic) finite state automaton, which is defined by the five-tuple $G:=\left(X, \Sigma, f, \Gamma, x_{0}\right)$ where $X$ is the finite set of states, $\Sigma$ is the finite set of events, $f: X \times \Sigma \rightarrow X$ is the transition function, partially defined over its domain, $\Gamma: X \rightarrow 2^{\Sigma}$ is the active event function, being defined as $\Gamma(x):=\{\sigma \in \Sigma: f(x, \sigma) !\}$, where $f(x, \sigma)$ ! means that $\exists y \in X$ such that $f(x, \sigma)=y$, and $x_{0} \in X$ is the initial state. The transition function can be extended to $f: X \times \Sigma^{*} \rightarrow X$, where $\Sigma^{*}$ represents the KleeneClosure (Cassandras and Lafortune, 2008) of $\Sigma$, by the following recursion: $f(x, \varepsilon)=x$, where $\varepsilon$ denotes the empty sequence, and $f(x, \sigma s)=f(f(x, \sigma), s)$, for all $\sigma \in \Sigma$ and $s \in \Sigma^{*}$. The language generated by $G$ is defined as $L(G):=\left\{s \in \Sigma^{*}: f\left(x_{0}, s\right) !\right\}$. The prefix-closure of a 
language $L$ is defined as $\bar{L}:=\left\{s \in \Sigma^{*}:\left(\exists t \in \Sigma^{*}\right)[s t \in L]\right\}$. $L$ is said to be prefix-closed if $L=\bar{L}$; thus $L(G)$ is prefixclosed. A language $L$ is said to be live if, for all $s \in L$, there exists $\sigma \in \Sigma$ such that $s \sigma \in L$. Given a sequence $s \in L$, then the post-language of $L$ after $s$ is the language $L / s:=\left\{t \in \Sigma^{*}: s t \in L\right\}$.

Given two event sets $\Sigma_{a}$ and $\Sigma_{b}$, where $\Sigma_{b} \subseteq \Sigma_{a}$, the natural projection $P_{a, b}: \Sigma_{a}^{*} \rightarrow \Sigma_{b}^{*}$ is defined as (Ramadge and Wonham, 1989): (i) $P_{a, b}(\varepsilon)=\varepsilon$; (ii) $P_{a, b}(\sigma)=\sigma$, if $\sigma \in \Sigma_{b}$, or $P_{a, b}(\sigma)=\varepsilon$, if $\sigma \in \Sigma_{a} \backslash \Sigma_{b}$, and; (iii) $P_{a, b}(s \sigma)=P_{a, b}(s) P_{a, b}(\sigma)$, where $s \in \Sigma_{a}^{*}$ and $\sigma \in \Sigma_{a}$. The inverse projection is defined as $P_{a, b}^{-1}: \Sigma_{b}^{*} \rightarrow 2^{\Sigma_{a}^{*}}$, where $P_{a, b}^{-1}(t):=\left\{s \in \Sigma_{a}^{*}: P_{a, b}(s)=t\right\}$. The projection and the inverse projection are extended over a language $L$ by applying them to each sequence of $L$.

Throughout the text, $\|s\|($ resp. $|B|)$ denotes the length (resp. cardinality) of sequence $s$ (resp. set $B$ ), and $s^{1}$ and $s / s^{1}$ denote, respectively, the first event of $s$ and the sequence obtained by removing $s^{1}$ from $s$. Given a set of events $\Sigma$ and a natural number $k, \Sigma^{(k)}$ denotes the set of sequences belonging to $\Sigma^{*}$ whose length is smaller or equal than $k$, i.e., $\Sigma^{(k)}=\left\{s \in \Sigma^{*}:\|s\| \leq k\right\}$. The accessible part of an automaton $G$ is denoted by $A c(G)$. For two automata $G_{1}$ and $G_{2}$, whose sets of events are, respectively, $\Sigma_{1}$ and $\Sigma_{2}$, the parallel composition between $G_{1}$ and $G_{2}$ is denoted by $G_{1} \| G_{2}$, and its generated language is $L\left(G_{1} \| G_{2}\right)=P_{1}^{-1}\left(L\left(G_{1}\right)\right) \cap P_{2}^{-1}\left(L\left(G_{2}\right)\right)$, where $P_{i}:\left(\Sigma_{1} \cup\right.$ $\left.\Sigma_{2}\right)^{*} \rightarrow \Sigma_{i}^{*}, i=1,2$ (Cassandras and Lafortune, 2008, sec.2.3).

We say that a set of states $B \subseteq X$ is a nontrivial strongly connected component (SCC) of $G$ if: (i) every pairs $x$ and $y$ in $B$ are reachable from each other, (ii) $B$ is maximal, in the sense that there is no other state that can be included in $B$ by satisfying the reachability condition stated in (i), and; (iii) if $B=\{x\}$ (i.e., $|B|=1$ ), then $\exists \sigma \in \Sigma: f(x, \sigma)=x$.

We consider that the event set of automaton $G$ is partitioned as $\Sigma=\Sigma_{o} \dot{\cup} \Sigma_{u o}$, where $\Sigma_{o}$ and $\Sigma_{u o}$ are, respectively, the sets of observable and unobservable events. The observer automaton (Cassandras and Lafortune, 2008, Sec. 2.5.2) of $G$ with respect to $\Sigma_{o}$, to be denoted by $\operatorname{Obs}\left(G, \Sigma_{o}\right)$, is a deterministic automaton whose generated language is $P_{o}(L(G))$, where $P_{o}: \Sigma^{*} \rightarrow \Sigma_{o}^{*}$.

Let $\Sigma_{f} \subset \Sigma_{u o}$ denote the set of failure events, which, for simplicity, is assumed to be a singleton, i.e., $\Sigma_{f}=\left\{\sigma_{f}\right\}$. In the problem of failure diagnosis using discrete event models, we want to be sure that the failure has actually occurred within a bounded number of steps after the occurrence of $\sigma_{f}$. Debouk et al. (2000) proposed a decentralized architecture, in which, local diagnosers $L D_{i}, i=1, \ldots, N$, partially observe the system behavior, being $\Sigma_{o, i} \subseteq \Sigma_{o}$ the set of observable events of $L D_{i}$. The communication between the local diagnosers and a coordinator, which makes the decision regarding the failure occurrence, is performed according to a predetermined rule, called protocol. One of the protocols proposed by Debouk et al. (2000) determines that each local diagnoser processes its observed sequence, and only communicates its diagnosis decision to the coordinator, which points out the failure occurrence if at least one local diagnoser has diagnosed it. Such a protocol has led to the concept of codiagnosability. In order to formally define codiagnosability, let $L=L(G)$. A sequence $s \in L$ is called a failure sequence if it has an event belonging to $\Sigma_{f}$. On the other hand, a sequence $s$ that has no failure event is referred to as a normal sequence. The language formed with all normal sequences is denoted by $L_{N} \subset L$, and, thus, the language formed with all failure sequences is equal to $L \backslash L_{N}$.

Definition 1. (Debouk et al., 2000). Assume that there are $N$ local diagnosers with projections $P_{o_{i}}: \Sigma \rightarrow \Sigma_{o, i}$, $i=1, \ldots, N$. Then, a prefix-closed and live language $L \subseteq \Sigma^{*}$ is codiagnosable with respect to $P_{o_{i}}, i=1, \ldots, N$, and $\Sigma_{f}$ if:

$$
\begin{gathered}
(\exists z \in \mathbb{N})\left(\forall s \in L \backslash L_{N}\right)(\forall t \in L / s,\|t\| \geq z) \Rightarrow \\
(\exists i \in\{1, \ldots, N\})\left[P_{o_{i}}(s t) \neq P_{o_{i}}(w), \forall w \in L_{N}\right] .
\end{gathered}
$$

The verification of codiagnosability can be carried out using test diagnosers (Debouk et al., 2000; Viana and Basilio, 2019). The procedure proposed by Viana and Basilio (2019) has advantages over that proposed by Debouk et al. (2000), because it does not require the assumption of the non-existence of cycles of states connected with unobservable events, and, additionally, it searches for nontrivial strongly connected components. In order to construct the test diagnoser proposed by Viana and Basilio (2019), we first define the label automaton $A_{\ell}=\left(X_{A_{\ell}},\left\{\sigma_{f}\right\}, f_{A_{\ell}}, \Gamma_{A_{\ell}}, x_{0, A_{\ell}}\right)$, where $X_{A_{\ell}}=\{N, Y\}$, $f_{A_{\ell}}\left(N, \sigma_{f}\right)=f_{A_{\ell}}\left(Y, \sigma_{f}\right)=Y$ and $x_{0, A_{\ell}}=N$. Next, we set $G_{\ell}=G \| A_{\ell}=\left(X_{\ell}, \Sigma, f_{\ell}, \Gamma_{\ell}, x_{0, \ell}\right)$, and, in the sequel, we compute, for $i=1, \ldots, N$,

$$
G_{d, i}=O_{b s}\left(G_{\ell}, \Sigma_{o, i}\right)=\left(X_{d_{i}}, \Sigma_{o, i}, f_{d_{i}}, \Gamma_{d_{i}}, x_{0, d_{i}}\right),
$$

which are the observer automata associated with local diagnosers $L D_{i}, i=1, \ldots, N$. An state of $G_{d, i}$ is said to be an uncertain state if it is formed with both $\mathrm{N}$ and $\mathrm{Y}-$ labeled states of $G_{\ell}$. Finally, we build the test diagnoser

$$
G_{S C C}=\left(\|_{i=1}^{N} G_{d, i}\right) \| G_{\ell} .
$$

Lemma 1. (Viana and Basilio, 2019). A language $L$ generated by an automaton $G$ is codiagnosable with respect to $P_{o_{i}}, i=1, \ldots, N$, and $\Sigma_{f}$ if, and only if, test diagnoser $G_{S C C}$ does not have any nontrivial SCC formed with states $\left(x_{d_{1}}^{1}, \ldots, x_{d_{N}}^{1}, x_{\ell}^{1}\right),\left(x_{d_{1}}^{2}, \ldots, x_{d_{N}}^{2}, x_{\ell}^{2}\right)$, $\ldots,\left(x_{d_{1}}^{m}, \ldots, x_{d_{N}}^{m}, x_{\ell}^{m}\right)$, such that, for all $j \in\{1, \ldots, m\}$, states $x_{d_{i}}^{j}, i=1, \ldots, N$, are uncertain and $x_{\ell}^{j}$ is an $\mathrm{Y}$ labeled state.

\section{STRUCTURE OF NETWORKED DISCRETE EVENT SYSTEMS SUBJECT TO DoS ATTACKS}

In this paper, we consider the system structure depicted in Figure 1, which is composed of a plant, modeled by an automaton $G=\left(X, \Sigma, f, \Gamma, x_{0}\right)$, a set of measurement sites $M S_{j}, j=1, \ldots, M$, a set of local diagnosers $L D_{i}$, $i=1, \ldots, N$, and a coordinator. Each measurement site $M S_{j}, j \in\{1, \ldots, M\}$, is able to record the occurrences of the observable events of the system belonging to set $\Sigma_{M S_{j}} \subseteq \Sigma_{o}$. In the case when there exists a communication channel $c h_{i, j}, i \in\{1, \ldots, N\}$ and $j \in\{1, \ldots, M\}$ that connects $M S_{j}$ to $L D_{i}$, then, channel $c h_{i, j}$ may be used to transmit some of the event occurrences recorded by $M S_{j}$ to $L D_{i}$, i.e., only events in $\Sigma_{M S_{j}}$ can be transmitted through 


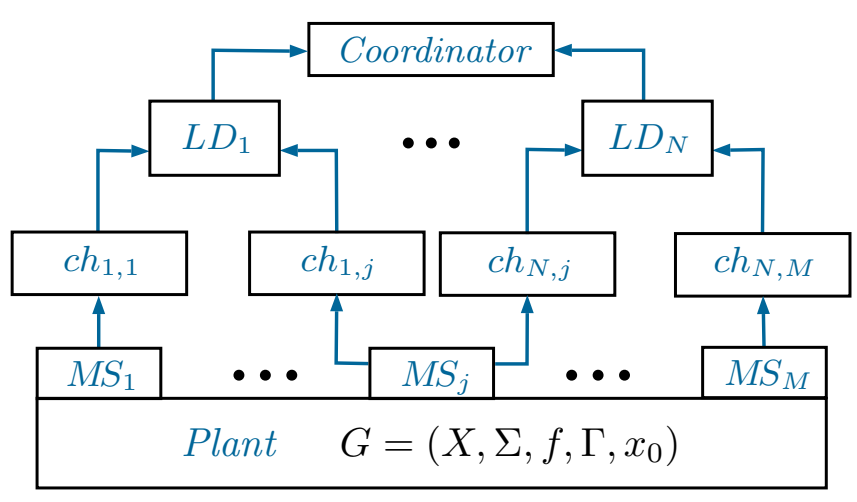

Figure 1. Decentralized diagnosis of NDES architecture.

some communication channel $c h_{i, j}, j \in\{1, \ldots, M\}$. We assume that there is at most one communication channel that connects a measurement site $M S_{j}$ to a local diagnoser $L D_{i}$. Each $L D_{i}$ only communicates its diagnosis decision to the coordinator, which points out a failure occurrence if at least one $L D_{i}, i \in\{1, \ldots, N\}$, has diagnosed it.

Let $\Sigma_{o_{i, j}}$ be the set of events whose occurrences are recorded by $M S_{j}, i \in\{1, \ldots, N\}$, and transmitted to $L D_{i}$, $j \in\{1, \ldots, M\}$. Then, we have that $\Sigma_{o_{i, j}} \subseteq \Sigma_{M S_{j}}$ and, in the case when channel $c h_{i, j}$ does not exist, $\Sigma_{o_{i, j}}=\emptyset$, so that we can obtain the set of locally observable events, from the point of view of $L D_{i}$, as follows:

$$
\Sigma_{o, i}=\bigcup_{j=1}^{M} \Sigma_{o_{i, j}}=\bigcup_{j \in J_{i}} \Sigma_{o_{i, j}},
$$

where $J_{i}=\left\{j \in\{1, \ldots, M\}: c h_{i, j}\right.$ exists $\}$ is the set of indices $j$ associated with the measurement sites $M S_{j}$ that have communication channels with local diagnoser $L D_{i}$.

The following assumptions are made:

A1 Every observable event in $\Sigma_{o}$ has its occurrences recorded by one, and only one, measurement site $M S_{j}, j \in\{1, \ldots, M\}$.

A2 Each communication channel $c h_{i, j}, i \in\{1, \ldots, N\}$ and $j \in J_{i}$, has a transmission rate that is known a priori to be equal to $r_{i, j}$ packets per step, where $r_{i} \in \mathbb{Z}_{+}^{*}\left(\mathbb{Z}_{+}^{*}\right.$ denotes the set of strictly positive integers).

A3 Each communication channel $c h_{i, j}, i \in\{1, \ldots, N\}$ and $j \in J_{i}$, has a bounded buffer that stores the packets to be transmitted, which is modeled by a firstin first-out (FIFO) queue, whose maximum length is known a priori be equal to $k_{i, j} \in \mathbb{Z}_{+}^{*}$.

A4 The set of vulnerable communication channels is know a priori.

A5 Language $L(G)$, generated by the plant, is live.

According to Assumption A1, $\Sigma_{M S_{m}} \cap \Sigma_{M S_{n}}=\emptyset$ for all $m, n \in\{1, \ldots, M\}$ such that $m \neq n$, which also implies that, $\Sigma_{o_{i, j}} \cap \Sigma_{o_{i, n}}=\emptyset$ for all $i \in\{1, \ldots, N\}$, $j, n \in\{1, \ldots, M\}$ and $j \neq n$. Assumption $\mathbf{A} 2$ implies that a communication channel $c h_{i, j}$ is able to transmit at most $r_{i}$ packets between two subsequent event occurrences in the plant. According to Assumption A3, the transmission through a communication channel $c h_{i, j}$ is FIFO, and the capacity of its buffer is equal to $k_{i, j}$ packets. As a consequence, all occurrences of events in $\Sigma_{o_{i, j}}$ recorded while the buffer of $c h_{i, j}$ is fully occupied will be discarded, hence, not being transmitted to $L D_{i}$. Assumption $\mathbf{A 4}$ implies that the communication channels are previously partitioned into two groups: (i) vulnerable communication channels, and (ii) secure communication channels; and so, we can define the sets of index pairs

$I_{v u l}=\left\{(i, j): i \in\{1, \ldots, N\} \wedge j \in J_{i} \wedge c h_{i, j}\right.$ is vulnerable $\}$ $I_{\text {sec }}=\left\{(i, j): i \in\{1, \ldots, N\} \wedge j \in J_{i} \wedge c h_{i, j}\right.$ is secure $\}$

associated with vulnerable and secure communication channels, respectively. Finally, it can be seen that Assumption A5 is not restrictive, since, if $L(G)$ is not live, we can obtain an automaton $G^{\prime}$ whose generated language $L\left(G^{\prime}\right)$ is live, by adding a new unobservable event to $G$ and adding self-loops, labeled by this event, at each state of $G$ that has no active event.

We consider that malicious agents are able to flood the buffers of the vulnerable communication channels $c h_{i, j}$, $(i, j) \in I_{v u l}$, with two types of false packets: (i) valid packets (with spoofed source addresses) that has a false event occurrence information, and (ii) meaningless packets that, although they are further discarded by some layer of the communication protocol, their processing may cause delay in the transmission of legitimate packets. Consequently, the malicious agents can carry out DoS attacks that generate false event observations and/or disrupt, temporally or definitely, the transmission of legitimate packets through vulnerable communication channels. Such adverse effects are illustrated in the following example.

Example 1. Consider the NDES depicted in Figure 2 whose plant is modeled by automaton $G$, where $\Sigma=$ $\left\{\alpha, \beta, \gamma, \delta, \mu, \sigma_{f}\right\}, \Sigma_{o}=\{\alpha, \beta, \gamma, \delta\}$ and $\Sigma_{f}=\left\{\sigma_{f}\right\}$. The system has also the following devices: (i) three measurement sites $M S_{1}, M S_{2}$ and $M S_{3}$ that record, respectively, the occurrences of the observable events in $\Sigma_{M S_{1}}=\{\beta\}$, $\Sigma_{M S_{2}}=\{\alpha\}$ and $\Sigma_{M S_{3}}=\{\gamma, \delta\}$; (ii) two local diagnosers, $L D_{1}$ and $L D_{2}$, and; (iii) four communication channels, where channels $c h_{1,1}$ and $c h_{1,2}$ (resp. $c h_{22}$ and $c h_{23}$ ) transmit the occurrences of the events in $\Sigma_{o_{1,1}}=\{\beta\}$ and $\Sigma_{o_{1,2}}=\{\alpha\}$ (resp. $\Sigma_{o_{2,2}}=\{\alpha\}$ and $\left.\Sigma_{o_{2,3}}=\{\gamma, \delta\}\right)$ to local diagnoser $L D_{1}$ (resp. $L D_{2}$ ). Thus, the sets of locally observable events of $L D_{1}$ and $L D_{2}$ are $\Sigma_{o, 1}=\{\beta, \alpha\}$ and $\Sigma_{o, 2}=\{\alpha, \gamma, \delta\}$, respectively. It can be checked that, by assuming no DoS attacks, language $L(G)$ is codiagnosable with respect to $P_{o_{i}}: \Sigma^{*} \rightarrow \Sigma_{o, i}^{*}, i=1,2$, and $\Sigma_{f}$.

Let us now consider the existence of malicious agents that are able to perform DoS attacks. In order to do so, we consider that $k_{1,1}=k_{1,2}=k_{2,3}=1$ and $r_{1,1}=r_{1,2}=$ $r_{2,3}=1$, and that communication channels $c h_{1,1}, c h_{1,2}$ and $c h_{2,3}$ are secure, i.e., $I_{\text {sec }}=\{(1,1),(1,2),(2,3)\}$. On the other hand, we assume that communication channel $c h_{2,2}$ is vulnerable to DoS attacks, i.e., $I_{v u l}=\{(2,2)\}$, and its buffer capacity and transmission rate are $k_{2,2}=2$ and $r_{2,2}=1$, respectively. Since channel $c_{2,2}$ is vulnerable, the malicious agent can insert packets into the buffer of this channel, which may contain either a false event occurrence of $\alpha$, represented here by event $\alpha_{i n_{2}}$, or a meaningless packet, represented here by event $t_{2,2}$. Although the meaningless packets are further discarded by the communication protocol, their processing spends time causing delays in the transmission through channel $c h_{2,2}$.

Let us consider the following cases: 


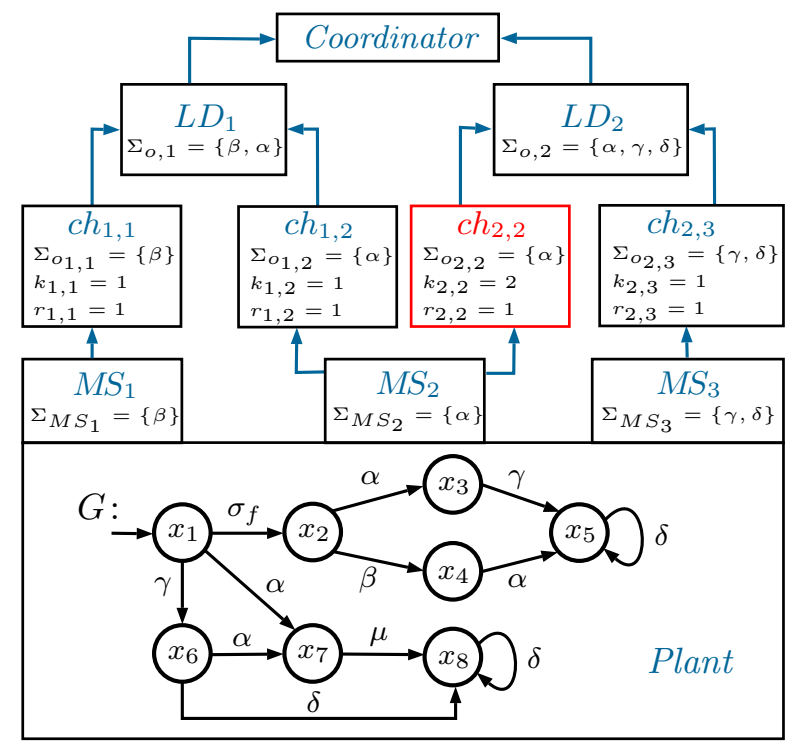

Figure 2. Networked DES considered in the running example.

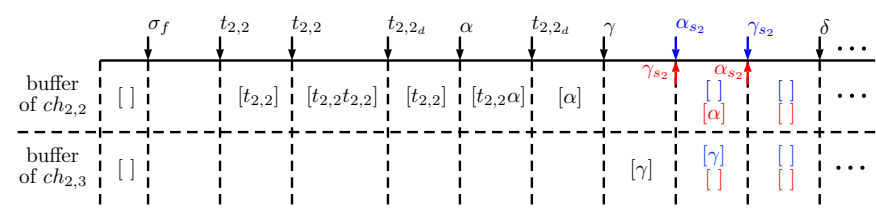

(a) Scenario (i)

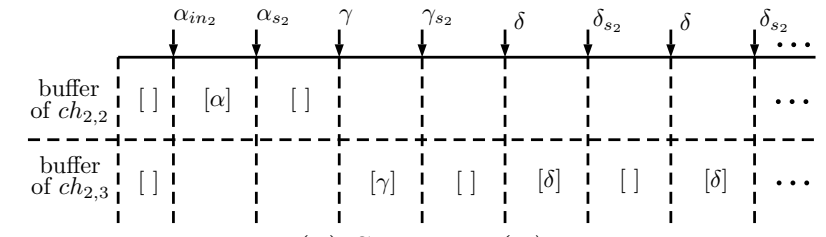

(b) Scenario (ii)

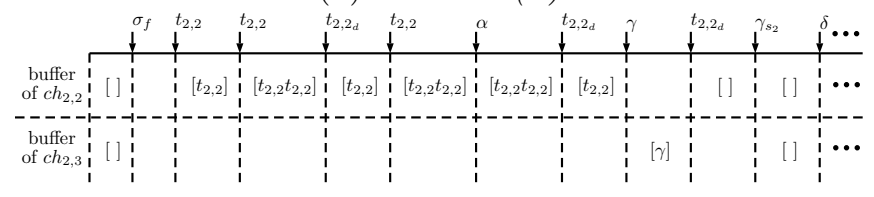

(c) Scenario (iii)

Figure 3. Event timelines of Example 1.

(i) Suppose that the plant has generated sequence $s_{1}=$ $\sigma_{f} \alpha \gamma \delta^{p}, p \in \mathbb{N}$, and the attacker has inserted, in $c h_{2,2}$, two meaningless packets $t_{2,2}$ after the occurrence of $\sigma_{f}$ but before the occurrence of $\alpha$. The completion of the processing of each packet $t_{2,2}$ is represented, in Figure 3(a), by event $t_{2,2_{d}}$. Since the transmission rate of channel $c h_{2,2}$ is one packet per step, the processing of the first packet $t_{2,2}$ finishes before the occurrence of event $\alpha$ but the processing of the second one finishes after $\alpha$, and, consequently, the successful transmission of $\alpha$ occurs only after the occurrence of $\gamma$, which means that the observation of $\alpha$ is delayed by one step. In addition, since the occurrences of $\alpha$ and $\gamma$ are transmitted through different communication channels to local diagnoser $L D_{2}$, the successful transmission of $\alpha$ and $\gamma$ to $L D_{2}$, represented by events $\alpha_{s_{2}}$ and $\gamma_{s_{2}}$ in Figure 3(a), may take place either (i.a) in the same order of occurrence in plant (the blue part in Figure 3(a)), or $(i . b)$ in an order different from that generated by the plant (the red part in Figure 3(a)). (ii) Suppose, now, that the plant has generated sequence $s_{2}=\gamma \delta^{p}, p \in \mathbb{N}$, and the attacker has inserted a packet with a false occurrence of event $\alpha$, represented by event $\alpha_{i n_{2}}$ in Figure 3(b), before the occurrence of $\gamma$. As seen from Figure 3(b), in this case, local diagnose $L D_{2}$ observes sequence $\alpha_{s_{2}} \gamma_{s_{2}} \delta_{s_{2}}^{p}$ instead of $\gamma_{s_{2}} \delta_{s_{2}}^{p}$, i.e., the malicious agent has created a false observation of event $\alpha$.

(iii) Suppose, again, that the plant has generated sequence $s_{1}=\sigma_{f} \alpha \gamma \delta^{p}, p \in \mathbb{N}$, and the attacker has inserted three meaningless packets $t_{2,2}$ between the occurrences of $\sigma_{f}$ and $\alpha$, as shown in the timeline of Figure 3(c). Notice from Figure 3(c) that, in this case, event $\alpha$ occurred in the plant while the buffer of channel $c h_{2,2}$ was fully occupied, and, consequently, the packet with the information of its occurrence was discarded due to the buffer overflow.

This example allows us to draw the following conclusions:

- A local diagnoser may observe the events in an order different from their actual order of occurrences in the plant, since a malicious agent may cause communication delays, by inserting meaningless packets into the buffers of vulnerable communication channels.

- A local diagnoser may observe false occurrences of events, since the malicious agents can insert valid packets with false event occurrences.

- The malicious agents can cause observation losses of the occurrences transmitted through vulnerable communication channels, by overflowing their buffers.

- Although DoS attacks may cause the loss of observation of an occurrence transmitted through a vulnerable channel $c h_{i, j}$, when its observation is not lost, its delay is, at most, equal to floor $\left(k_{i, j} / r_{i, j}\right)$, if $k_{i, j} / r_{i, j} \notin \mathbb{Z}_{+}^{*}$, and equal to $\left(k_{i, j} / r_{i, j}\right)-1$, if $k_{i, j} / r_{i, j} \in$ $\mathbb{Z}_{+}^{*}$. In addition, the maximal number of packets that a malicious agent can insert in the buffer of channel $c h_{i, j}$, between two consecutive event occurrences in the plant, is equal to $\left(k_{i, j}+r_{i, j}\right)$.

In this paper, we will address the problem of verifying if it is possible to detect the occurrence of failure events in the presence of DoS attacks. To do so, we propose, in the next section, a model for NDES subject to DoS attacks.

\section{AUTOMATON MODEL FOR NDES SUBJECT TO DoS ATTACKS}

In this section, we first introduce a procedure to construct automata $C_{i, j}$ that model each communication channel separately, and so, we compute, from these automata, automata $G_{n, i}$ that model the local communication networks used to transmit the event occurrences to each local diagnoser $L D_{i}, i=1, \ldots, N$. In the sequel, we present automaton $G_{a, i}$ that models a NDES subject to DoS attacks from the point of view of local diagnoser $L D_{i}$.

\subsection{Automaton models for communication channels}

In order to propose a systematic way for the construction of an automaton $C_{i, j}=\left(X_{i, j}, \Sigma_{i, j}, f_{i, j}, \Gamma_{i, j}, x_{0_{i, j}}\right)$ that models a communication channel $c h_{i, j}, i \in\{i, \ldots, N\}$ and $j \in J_{i}$, we need to take into account if $c h_{i, j}$ is either vulnerable (i.e., $\left.(i, j) \in I_{v u l}\right)$ or secure (i.e., $\left.(i, j) \in I_{\text {sec }}\right)$. In the case when $c h_{i, j}$ is vulnerable, the malicious agent 
can insert both, meaningless packets and valid packets with false event occurrence information, in the buffer of $c h_{i, j}$.

In order to deal with the insertion of valid packets, we distinguish a real occurrence of an event $\sigma \in \Sigma_{o_{i, j}},(i, j) \in$ $I_{v u l}$, from the insertion into $c h_{i, j}$ of a valid packet with the false information of an occurrence of $\sigma$, by representing the latter as a different event, to be denoted by $\sigma_{i n_{i}}$. Notice that, when the real occurrence of $\sigma$ or its false version $\sigma_{i n_{i}}$ are received by local diagnoser $L D_{i}$, they look the same, and, thus, we represent the successful transmission of both, $\sigma$ and $\sigma_{i n_{i}}$, by using the same event $\sigma_{s_{i}}{ }^{1}$

Regarding the insertion of meaningless packets in the buffer of channel $c h_{i, j},(i, j) \in I_{v u l}$, it is reasonable to consider that no information is received by local diagnoser $L D_{i}$ when a meaningless packet is transmitted through $c h_{i, j}$, since this packet is, in general, discarded by some layer of the communication protocol. However, the processing of this packet spends time, and consequently, the transmission of a future event occurrence $\sigma \in \Sigma_{o_{i, j}}$ may be delayed or even be discarded. In order to consider such effects, we define events $t_{i, j}$ and $t_{i, j_{d}}$ that model, respectively, the insertion of a meaningless packet in the buffer of channel $c h_{i, j}$ (by a malicious agent) and the completion of its processing. Notice that both events, $t_{i, j}$ and $t_{i, j_{d}}$, are unobservable to local diagnoser $L D_{i}$.

We may, now, define the set of events of automaton $C_{i, j}$, as follows:

$$
\Sigma_{i, j}:=\Sigma \dot{U} \Sigma_{i n_{i, j}} \dot{\cup} \Sigma_{s_{i, j}} \dot{\cup} \Sigma_{d_{i, j}},
$$

where set $\Sigma_{i n_{i, j}}$ is formed by the events that model the packet insertions into channel $c h_{i, j}$, set $\Sigma_{s_{i, j}}$ is formed by the events that model the successful transmissions through channel $c h_{i, j}$, and set $\Sigma_{d_{i, j}}$ has the event that represents the completion of the processing of a meaningless packet inserted into the buffer of channel $c h_{i, j}$, which are defined as follows:

$$
\begin{aligned}
& \Sigma_{i n_{i, j}}:= \begin{cases}\left\{\sigma_{i n_{i}}: \sigma \in \Sigma_{o_{i, j}}\right\} \dot{\cup}\left\{t_{i, j}\right\}, & \text { if }(i, j) \in I_{v u l} \\
\emptyset, & \text { if }(i, j) \in I_{\text {sec }}\end{cases} \\
& \Sigma_{s_{i, j}}:=\left\{\sigma_{s_{i}}: \sigma \in \Sigma_{o_{i, j}}\right\} \\
& \Sigma_{d_{i, j}}:= \begin{cases}\left\{t_{i, j_{d}}\right\}, & \text { if }(i, j) \in I_{v u l} \\
\emptyset, & \text { if }(i, j) \in I_{\text {sec }} .\end{cases}
\end{aligned}
$$

Notice that, for a secure communication channel $c h_{i, j}$, where $(i, j) \in I_{\text {sec }}$, the set of events $\Sigma_{i, j}$ becomes $\Sigma_{i, j}=$ $\Sigma \dot{\cup} \Sigma_{s_{i, j}}$ since $\Sigma_{i n_{i, j}}=\Sigma_{d_{i, j}}=\emptyset$.

Example 2. Consider the same NDES addressed in Example 1, which is depicted in Figure 2. Since $(2,2) \in$ $I_{v u l}$, for communication channel $c h_{2,2}$, we have $\Sigma_{i n_{2,2}}=$ $\left\{\alpha_{i n_{2}}, t_{2,2}\right\}, \Sigma_{s_{2,2}}=\left\{\alpha_{s_{2}}\right\}$ and $\Sigma_{d_{2,2}}=\left\{t_{2,2}\right\}$. On the other hand, for channel $c h_{2,3}$, we have that $\Sigma_{s_{2,3}}=$ $\left\{\gamma_{s_{2}}, \delta_{s_{2}}\right\}$ and $\Sigma_{i n_{2,3}}=\Sigma_{d_{2,3}}=\emptyset$ since $(2,3) \in I_{\text {sec }}$. Therefore, $\Sigma_{2,2}=\left\{\alpha, \alpha_{i n_{2}}, \alpha_{s_{2}}, t_{2,2}, t_{2,2_{d}}\right\}$ and $\Sigma_{2,3}=$ $\left\{\gamma, \delta, \gamma_{s_{2}}, \delta_{s_{2}}\right\}$.

Notice that Example 1 suggests that the effects of DoS attacks on the observations of local diagnoser $L D_{2}$ can be

\footnotetext{
1 We adopt notation $\sigma_{i n_{i}}\left(\operatorname{resp} . \sigma_{s_{i}}\right)$ instead of $\sigma_{i n_{i, j}}\left(\operatorname{resp} . \sigma_{s_{i, j}}\right)$ for simplicity, since, according to Assumption A1, the occurrences of an event $\sigma \in \Sigma_{o}$ are recorded by just one measurement site $M S_{j}$, namely, event $\sigma$ is recorded by the measurement site $M S_{j}$ such that $\sigma \in \Sigma_{M S_{j}}$
}

represented by sequences defined over $\left(\Sigma_{2,2} \cup \Sigma_{2,3}\right)$. For instance, sequences $w_{a}^{\prime}=\sigma_{f} t_{2,2} t_{2,2} t_{2,2_{d}} \alpha t_{2,2} \gamma \alpha_{s_{2}} \gamma_{s_{2}}\left(\delta \delta_{s_{2}}\right)^{p}$ and $w_{b}^{\prime}=\sigma_{f} t_{2,2} t_{2,2} t_{2,2_{d}} \alpha t_{2,2_{d}} \gamma \gamma_{s_{2}} \alpha_{s_{2}}\left(\delta \delta_{s_{2}}\right)^{p}$ model cases (i.a) and (i.b) described in Example 1, respectively. Moreover, sequences $w^{\prime \prime}=\alpha_{i n_{2}} \alpha_{s_{2}} \gamma \gamma_{s_{2}}\left(\delta \delta_{s_{2}}\right)^{p}$ and $w^{\prime \prime \prime}=$ $\sigma_{f} t_{2,2} t_{2,2} t_{2,2} t_{2,2} \alpha t_{2,2_{d}} \gamma t_{2,2_{d}} \gamma_{s_{2}}\left(\delta \delta_{s_{2}}\right)^{p}$ model cases (ii) and (iii) of Example 1, respectively.

Let us define two functions associated with a channel $c h_{i, j}$ :

- Function $\varphi_{i n_{i, j}}: \Sigma_{i n_{i, j}} \rightarrow\left(\Sigma_{o_{i, j}} \cup\left\{t_{i, j}\right\}\right)$ is a mapping defined as: (i) for all $\sigma_{i n_{i}} \in\left(\Sigma_{i n_{i, j}} \backslash\left\{t_{i, j}\right\}\right)$, $\varphi_{i n_{i, j}}\left(\sigma_{i n_{i}}\right)=\sigma$, and $(i i) \varphi_{i n_{i, j}}\left(t_{i, j}\right)=t_{i, j}$.

- Function $\varphi_{s_{i, j}}:\left(\Sigma_{o_{i, j}} \cup\left\{t_{i, j}\right\}\right) \rightarrow\left(\Sigma_{s_{i, j}} \cup \Sigma_{d_{i, j}}\right)$ is a mapping defined as: (i) for all $\sigma \in \Sigma_{o_{i, j}}, \varphi_{s_{i}}(\sigma)=\sigma_{s_{i}}$, and (ii) $\varphi_{s_{i}}\left(t_{i, j}\right)=t_{i, j_{d}}$.

In order to define the states of automaton $C_{i, j}$, we must take into account the state of the buffer of channel $c h_{i, j}$, and the amount of packet transmissions that can be performed through channel $c h_{i, j}$ until a new event occurrence in the plant, i.e., in the current step. We represent the state of the buffer of channel $c h_{i, j}$ by using a sequence $b \in\left(\Sigma_{o_{i, j}} \cup\left\{t_{i, j}\right\}\right)^{\left(k_{i, j}\right)}$ formed with those events associated with the packets stored by it. For example, by considering $(i, j) \in I_{v u l}, \alpha, \beta \in \Sigma_{o_{i, j}}$ and $\mu \in \Sigma \backslash \Sigma_{o_{i, j}}$, then, after the occurrence of sequence $s=\alpha \beta_{i n_{i}} \mu t_{i, j}$, the state of the buffer of channel $c h_{i, j}$ can be represented as $b=\alpha \beta t_{i, j}$ where $\alpha, \beta$ and $t_{i, j}$ represent, respectively, the packets inserted in the buffer of channel $c h_{i, j}$ due to the occurrences of $\alpha, \beta_{i n_{i}}$ and $t_{i, j}$. Notice that we insert $\beta$ instead of $\beta_{i n_{i}}$ in sequence $b$, because the contents of the packets associated with both, the false event occurrence $\beta_{i n_{i}}$ and the true one $\beta$, are the same. On the other hand, we do not include $\mu$ in $b$ since its occurrence inserts no packet in the buffer of channel $c h_{i, j}$. It is important to remark that $0 \leq\|b\| \leq k_{i, j}$, where $\|b\|=0$ (i.e., $b=\varepsilon$ ) when there is no event in the buffer of channel $c h_{i, j}$ and $\|b\|=k_{i, j}$ when it is fully occupied. Regarding the amount of packet transmissions that can be performed through channel $c h_{i, j}$ in the current step, it can be represented by an integer number $c \in \mathbb{Z}_{+}$, where $0 \leq c \leq r_{i, j}$ since channel $c h_{i, j}$ is able to transmit at most $r_{i, j}$ packets per step. We may, now, present the following results.

Lemma 2. Given a NDES where $L(G)=\Sigma^{*}$ and $M S_{m}$, $m \in\{1, \ldots, M\}$, is the unique measurement site that sends event occurrences to local diagnoser $L D_{i}$, i.e., $J_{n}=$ $\{m\}$ and $\Sigma_{o_{i, m}} \neq \emptyset$. Consider that the current state of the buffer of channel $c h_{i, m}$ is represented by sequence $b \in\left(\Sigma_{o_{i, j}} \cup\left\{t_{i, j}\right\}\right)^{\left(k_{i, j}\right)}$, and that the current amount of packet transmissions that can be performed through channel $c h_{i, m}$, until a new event occurrence in the plant, is equal to $c \in \mathbb{Z}_{+}$. Then,

(i) An event $\sigma \in \Sigma$ is currently able to occur if, and only if, $[(c=0) \vee(b=\varepsilon)]$.

(ii) An event $\sigma \in \Sigma_{i n_{i, m}}$ is currently able to occur if, and only if, $\|b\|<k_{i, m}$.

(iii) An event $\sigma \in\left(\Sigma_{s_{i, m}} \cup \Sigma_{d_{i, j}}\right)$ is currently able to occur if, and only if, $\left[(b \neq \varepsilon) \wedge\left(\sigma=\varphi_{s_{i, m}}\left(b^{1}\right)\right) \wedge(c \neq 0)\right]$.

Lemma 3. Let $\sigma \in \Sigma_{i, j}$ be an event that is currently able to occur for given $b \in\left(\Sigma_{o_{i, j}} \cup\left\{t_{i, j}\right\}\right)^{\left(k_{i, j}\right)}$ and $c \in \mathbb{Z}_{+}$, 
and let $b_{n e w}$ and $c_{n e w}$ denote, respectively, the state of the buffer of channel $c h_{i, m}$ and the amount of packet transmissions that can be performed through channel $c h_{i, m}$, until a new event occurrence in the plant, after the occurrence of $\sigma$. Then,

(i) If $\sigma \in \Sigma$, then $c_{\text {new }}=r_{i, m}$, and $b_{\text {new }}=b \sigma$, if $\left[\left(\sigma \in \Sigma_{o_{i, m}}\right) \wedge\left(\|b\|<k_{i, m}\right)\right]$, or $b_{\text {new }}=b$, otherwise.

(ii) If $\sigma \in \Sigma_{i n_{i, m}}$, then $b_{\text {new }}=b \varphi_{i n_{i, m}}(\sigma)$ and $c_{\text {new }}=c$.

(iii) If $\sigma \in\left(\Sigma_{s_{n, m}} \cup \Sigma_{d_{i, m}}\right)$, then $b_{n e w}=b / b^{1}$ and $c_{\text {new }}=(c-1)$.

Based on Lemmas 2 and 3, we can present the following automaton model for communication channels.

Definition 2. Automaton $C_{i, j}$ that models the communication channel $c h_{i, j}, i \in\{1, \ldots, N\}$ and $j \in J_{i}$, is defined as:

$$
\begin{aligned}
C_{i, j} & :=A c\left(Q_{i, j}, \Sigma_{i, j}, f_{i, j}, \Gamma_{i, j}, x_{0_{i, j}}\right) \\
& =\left(X_{i, j}, \Sigma_{i, j}, f_{i, j}, \Gamma_{i, j}, x_{0_{i, j}}\right),
\end{aligned}
$$

where: (i) $Q_{i, j}=\left(\Sigma_{o_{i, j}} \cup\left\{t_{i, j}\right\}\right)^{\left(k_{i, j}\right)} \times\left\{0,1, \ldots, r_{i, j}\right\}$; (ii) $\Sigma_{i, j}$ is defined in accordance with Eqs. (3a) to (3d); (iii) for all $(b, c) \in Q_{i, j}$ :

$$
\begin{aligned}
& \Gamma_{i, j}((b, c)):= \\
& \begin{cases}\Sigma \cup \Sigma_{i n_{i, j}}, & \text { if }\left[(c=0) \wedge\left(\|b\|<k_{i, j}\right)\right] \\
\Sigma, & \vee[(c \neq 0) \wedge(b=\varepsilon)], \\
\Sigma_{i n_{i, j}} \cup\left\{\varphi_{s_{i, j}}\left(b^{1}\right)\right\}, & \text { if }\left[(c \neq 0) \wedge\left(\|b\|=k_{i, j}\right)\right], \\
\left\{\varphi_{s_{i, j}}\left(b^{1}\right)\right\}, & \text { if }\left[(c \neq 0) \wedge\left(0<\|b\|<k_{i, j}\right)\right], \\
\emptyset, & \text { otherwise; }\end{cases}
\end{aligned}
$$

(iv) for all $(b, c) \in Q_{i, j}$ and $\sigma \in \Gamma_{i, j}((b, c))$ :

$$
\begin{aligned}
& f_{i, j}((b, c), \sigma):= \\
& \begin{cases}\left(b \sigma, r_{i, j}\right), & \text { if }\left[\left(\sigma \in \Sigma_{o_{i, j}}\right) \wedge\left(\|b\|<k_{i, j}\right)\right], \\
\left(b, r_{i, j}\right), & \text { if }\left[\left(\sigma \in \Sigma \backslash \Sigma_{o_{i, j}}\right) \vee\left(\|b\|=k_{i, j}\right)\right], \\
\left(b \varphi_{i n_{i, j}}(\sigma), c\right), & \text { if } \sigma \in \Sigma_{i n_{i, j}}, \\
\left(b / b^{1}, c-1\right), & \text { if } \sigma \in\left(\Sigma_{s_{i, j}} \cup \Sigma_{d_{i, j}}\right) ;\end{cases}
\end{aligned}
$$

(v) $x_{0_{i, j}}=\left(\varepsilon, r_{i, j}\right)$.

The construction of the communication channel automaton $C_{i, j}$ can be performed in accordance with Algorithm 1.

Algorithm 1. (Construction of automaton $C_{i, j}$ ).

Inputs: Set $\Sigma$ of the plant events, set $\Sigma_{o_{i, j}}$ formed with the events whose occurrences are transmitted through channel $c h_{i, j}$, and the buffer capacity $k_{i, j}$ and the transmission rate $r_{i, j}$ of channel $c h_{i, j}$.

Output: Automaton $C_{i, j}=\left(X_{i, j}, \Sigma_{i, j}, f_{i, j}, \Gamma_{i, j}, x_{0_{i, j}}\right)$.

STEP 1. Define $x_{0_{i, j}}=\left(\varepsilon, r_{i, j}\right)$ and $X_{i, j}=\left\{x_{0_{i, j}}\right\}$.

STEP 2. Build sets $\Sigma_{i, j}, \Sigma_{i n_{i, j}}, \Sigma_{s_{i, j}}$ and $\Sigma_{d_{i, j}}$ according to Eqs. (3a) to (3d).

Step 3. Create a FIFO queue $F=\left[x_{0_{i, j}}\right]$.

SteP 4. While $F \neq \emptyset$ do:

4.1. $(b, c) \leftarrow \operatorname{head}(F)$ and dequeue $(F)$.

4.2. If $(c=0) \vee(b=\varepsilon)$, then, for all $\sigma \in \Sigma$ :
4.2.1. Define:

$$
\tilde{x}_{\sigma}=f_{i, j}((b, c), \sigma)= \begin{cases}\left(b \sigma, r_{i, j}\right), & \text { if }\left(\sigma \in \Sigma_{o_{i, j}}\right) \\ & \wedge\left(\|b\|<k_{i, j}\right) \\ \left(b, r_{i, j}\right), & \text { otherwise. }\end{cases}
$$

4.2.2. If $\tilde{x}_{\sigma} \notin X_{i, j}$, then set $X_{i, j} \leftarrow X_{i, j} \cup\left\{\tilde{x}_{\sigma}\right\}$ and enqueue $\left(F, \tilde{x}_{\sigma}\right)$.

4.3. If $\|b\|<k_{i, j}$, then, for all $\sigma \in \Sigma_{i n_{i, j}}$ :

4.3.1. Define $\hat{x}_{\sigma}=f_{i, j}((b, c), \sigma)=\left(b \varphi_{i n_{i, j}}(\sigma), c\right)$.

4.3.2. If $\hat{x}_{\sigma} \notin X_{i, j}$, then set $X_{i, j} \leftarrow X_{i, j} \cup\left\{\hat{x}_{\sigma}\right\}$ and enqueue $\left(F, \hat{x}_{\sigma}\right)$.

4.4. If $(c \neq 0) \wedge(\|b\| \neq 0)$, then:

4.4.1. Define $\bar{x}=f_{i, j}\left((b, c), \varphi_{s_{i, j}}\left(b^{1}\right)\right)=\left(b / b^{1}, c-1\right)$.

4.4.2. If $\bar{x}_{\sigma} \notin X_{i, j}$, then set $X_{i, j} \leftarrow X_{i, j} \cup\left\{\bar{x}_{\sigma}\right\}$ and enqueue $\left(F, \bar{x}_{\sigma}\right)$.

STEP 5. Define $\Gamma_{i, j}(x)=\left\{\sigma \in \Sigma_{i, j}: f_{i, j}(x, \sigma) !\right\}$, for all $x \in X_{i, j}$.

Example 3. Consider the communication channel $c h_{2,2}$ of the NDES depicted in Figure 2. As in Example 1, it is assumed that $c h_{2,2}$ is vulnerable to DoS attacks. Automaton $C_{2,2}$, constructed in accordance with Algorithm 1, is depicted in Figure 4. Notice, from Figure 4, that all augmented sequences presented in Example 2, $w_{a}^{\prime}, w_{b}^{\prime}, w^{\prime \prime}$ and $w^{\prime \prime \prime}$, belong to $L\left(C_{2,2}\right)$.

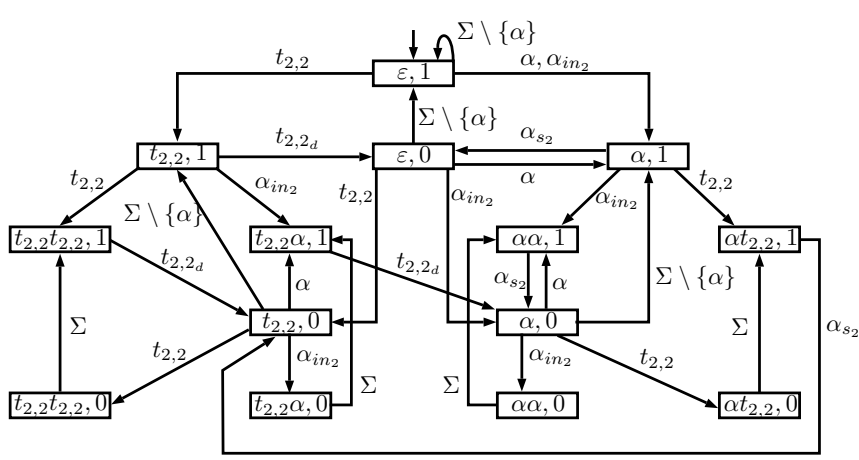

Figure 4. Automaton $C_{2,2}$ that models the vulnerable channel $c h_{2,2}$.

\subsection{Models for local communication networks and NDES subject to DoS attacks}

Given a local diagnoser $L D_{i}, i \in\{1, \ldots, N\}$, once we have automata $C_{i, j}$ for every $j \in J_{i}$, we can obtain an automaton $G_{n, i}$ that models the local communication network that is used to transmit the event occurrences to $L D_{i}$, as follows:

$$
G_{n, i}:=\|_{j \in J_{i}} C_{i, j}=\left(X_{n, i}, \Sigma_{a, i}, f_{n, i}, \Gamma_{n, i}, x_{0_{n, i}}\right) .
$$

It can be seen that the augmented event set $\Sigma_{a, i}$ of automaton $G_{n, i}$ is partitioned as $\Sigma_{a, i}=\Sigma \dot{\cup} \Sigma_{i n, i} \dot{\cup} \Sigma_{s, i} \dot{\cup} \Sigma_{d, i}$, where $\Sigma_{i n, i}=\bigcup_{j \in J_{i}} \Sigma_{i n_{i, j}}, \Sigma_{s, i}=\bigcup_{j \in J_{i}} \Sigma_{s_{i, j}}$ and $\Sigma_{d, i}=$ $\bigcup_{j \in J_{i}} \Sigma_{d_{i, j}}$. Notice that the new set of locally observable events of local diagnoser $L D_{i}$ is $\Sigma_{s, i}$. Let us define the following projections:

$$
P_{a_{i}}: \Sigma_{a, i}^{*} \rightarrow \Sigma^{*}
$$




$$
P_{a_{i}, s_{i}}: \Sigma_{a, i}^{*} \rightarrow \Sigma_{s, i}^{*} .
$$

The following results concern the language generated by automaton $G_{n, i}$.

Lemma 4. The following statements hold true:

(a) Every sequence $w \in L\left(G_{n, i}\right)$ represents the case when sequence $s=P_{a_{i}}(w)$ is generated by the plant, and local diagnoser $L D_{i}$ observes sequence $s_{s_{i}}=P_{a_{i}, s_{i}}(w)$.

(b) For every sequence $s \in \Sigma^{*}, L\left(G_{n, i}\right)$ contains all augmented strings $w \in \Sigma_{a, i}^{*}$ that represent the occurrence of $s$ in the presence of DoS Attacks.

Lemma 4 suggests that automaton $G_{n, i}$ can be used to model the observation of local diagnoser $L D_{i}$, when the plant generates any sequence $s \in \Sigma^{*}$, in the presence of DoS attacks. Thus, based on language $L\left(G_{n, i}\right)$, we introduce the following language function.

Definition 3. (attack augmentation function). The attack augmentation function is the mapping $F_{a, i}: 2^{\Sigma^{*}} \rightarrow 2^{\Sigma_{a, i}^{*}}$, where $F_{a, i}(L):=P_{a_{i}}^{-1}(s) \cap L\left(G_{n, i}\right)$.

Let us also define the following automaton:

$$
G_{a, i}:=G \| G_{n, i}=\left(X_{a, i}, \Sigma_{a, i}, f_{a, i}, \Gamma_{a, i}, x_{0_{a, i}}\right) .
$$

We may, now, present the following result.

Theorem 1. Given a NDES subject to DoS attacks, where language $L(G) \subseteq \Sigma^{*}$ is live and local diagnoser $L D_{i}$ receives event occurrences sent by every measurement site $M S_{j}$ such that $j \in J_{i}$. Then, the augmented language $F_{a, i}(L(G))$ is formed by those sequences that model the effects of DoS attacks on the observation of local diagnoser $L D_{i}$. Moreover, $L\left(G_{a, i}\right)=F_{a, i}(L(G))$.

\section{ROBUST CODIAGNOSABILITY OF NDES SUBJECT TO DoS ATTACKS}

\subsection{Definition of robust codiagnosability of NDES}

Based on the modeling for NDES subject to DoS attacks presented in Section 4, we introduce, now, the concept of robust codiagnosability in the presence of DoS attacks.

Definition 4. (DoS-robust codiagnosability). A live and prefix-closed language $L \subseteq \Sigma^{*}$ is DoS-robustly codiagnosable with respect to $F_{a, i}, P_{a_{i}, s_{i}}, i=1, \ldots, N$, and $\Sigma_{f}$ if:

$$
(\exists z \in \mathbb{N})\left(\forall s \in L \backslash L_{N}\right)(\forall t \in L / s,\|t\| \geq z) \Rightarrow
$$$$
(\exists i \in\{1, \ldots, N\})\left[P_{a_{i}, s_{i}}\left(F_{a, i}(s t)\right) \cap P_{a_{i}, s_{i}}\left(F_{a, i}(w)\right)=\emptyset,\right.
$$$$
\left.\forall w \in L_{N}\right] \text {. }
$$

According to Definition 4, a live and prefix-closed language $L$ is not DoS-robustly codiagnosable if there exist a failure sequence $s$ and an arbitrarily long sequence $t$, such that there exist sequences $s_{a_{i}} t_{a_{i}} \in F_{a, i}(s t), i=1, \ldots, N$, and sequences $w_{a_{i}} \in F_{a, i}\left(w_{i}\right)$, with $w_{i} \in L_{N}$, satisfying $P_{a_{i}, s_{i}}\left(s_{a_{i}} t_{a_{i}}\right)=P_{a_{i}, s_{i}}\left(w_{a_{i}}\right)$, and so, DoS attacks may create ambiguous observations for all local diagnosers.

\subsection{Verification of robust codiagnosability of NDES}

In order to verify whether the language generated by a NDES subject to DoS attacks is DoS-robustly codiagnosable, we propose a test diagnoser, based on that proposed by Viana and Basilio (2019) for the codiagnosability verification. In order to do so, set $G_{\ell}:=G \| A_{\ell}$, where $G$ is the plant automaton and $A_{\ell}$, as defined in Section 2, is the label automaton. Notice that $L\left(G_{\ell}\right)=L(G)$. Thus, according to Theorem 1, we can obtain automata that model the NDES in the presence of DoS attacks, from the point of view of local diagnosers $L D_{i}, i=1, \ldots, N$, as follows:

$$
G_{a, i}^{\ell}:=G_{\ell} \| G_{n, i} .
$$

Moreover, their observer automata over $\Sigma_{s, i}, i=1, \ldots, N$, are

$$
G_{d, i}^{r o b}:=\operatorname{Obs}\left(G_{a, i}^{\ell}, \Sigma_{s, i}\right) .
$$

In order to illustrate the idea behind the verification of the DoS-robust diagnosability condition, consider the case when each local diagnoser $L D_{i}$ is unable to detect a unique failure sequence $s_{a_{i}} t_{a_{i}} \in L\left(G_{a, i}^{\ell}\right)$, or, equivalently, $L D_{i}$ is unable to detect the failure sequence $s_{i} t_{i}=P_{a_{i}}\left(s_{a_{i}} t_{a_{i}}\right) \in$ $L(G)$ in the presence of DoS attacks. In this case, according to Lemma 1 (Viana and Basilio, 2019), automaton $G_{S C C, i}=G_{d, i}^{r o b} \| G_{a, i}^{\ell}$ has a nontrivial SCC formed with states $\left(x_{d_{i}}^{1}, x_{\ell_{i}}^{1}\right), \ldots,\left(x_{d_{i}}^{m}, x_{\ell_{i}}^{m}\right)$, such that, for all $p \in$ $\{1, \ldots, m\}$, state $x_{d_{i}}^{p}$ is uncertain and $x_{\ell_{i}}^{p}$ is an Y-labeled state. However, we must check if every local diagnoser $L D_{i}$ is unable to detect the same arbitrarily long failure sequence, i.e., $s_{i} t_{i}=s t \in L(G)$, for all $i \in\{1, \ldots, N\}$. In order to do so, we define the DoS-robust test diagnoser as follows ${ }^{2}$ :

$$
G_{S C C}^{r o b}:=\left(\|_{i=1}^{N} G_{d, i}^{r o b}\right)\left\|G_{\ell}\right\|\left(\|_{i=1}^{N} G_{n, i}\right) .
$$

Fact 1. Let $P_{a, a_{i}}:\left(\bigcup_{i=1}^{N} \Sigma_{a, i}\right)^{*} \rightarrow \Sigma_{a, i}^{*}$. Then, $L\left(G_{S C C}^{r o b}\right)=$ $\bigcap_{i=1}^{N} P_{a, a_{i}}^{-1}\left(F_{a, i}(L(G))\right)$.

Theorem 2. Let $L$ be a live language generated by an automaton $G$. Consider the DoS-robust test diagnoser $G_{S C C}^{r o b}$ stated by Eq. (6). Then, $L$ is DoS-robustly codiagnosable with respect to $F_{a, i}$ and $P_{a_{i}, s_{i}}, i=1, \ldots, N$, and $\Sigma_{f}$ if, and only if, $G_{S C C}^{r o b}$ has no nontrivial SCC formed with states $\left(x_{d}^{1}, x_{\ell}^{1}, x_{n}^{1}\right), \ldots,\left(x_{d}^{m}, x_{\ell}^{m}, x_{n}^{m}\right)$, such that, for all $p \in\{1, \ldots, m\}, x_{\ell}^{p}$ is an Y-labeled state, and $x_{d}^{p}=\left(x_{d}^{p, 1}, \ldots, x_{d}^{p, N}\right)$, where states $x_{d}^{p, i}, i=1, \ldots, N$, are uncertain states.

Based on Theorem 2, we develop Algorithm 2.

Algorithm 2. (DoS-robust codiagnosability verification).

Inputs: Plant automaton $G$, the local communication network automata $G_{n, i}$ and the new sets of locally observable events $\Sigma_{s, i}$ for $i=1, \ldots, N$.

Output: Codiagnosability decision: True or False.

STEP 1. Compute the DoS-robust test diagnoser $G_{S C C}^{r o b}=\left(\|_{i=1}^{N} G_{d, i}^{r o b}\right)\left\|G_{\ell}\right\|\left(\|_{i=1}^{N} G_{n, i}\right)$.

STEP 2. Find all nontrivial SCC of $G_{S C C}^{r o b}$.

\footnotetext{
2 Notice that, as opposed to test diagnoser $G_{S C C}$ (Eq.(1)) where automata $G_{d, i}, i=1, \ldots, N$, are computed from the same automaton $G_{\ell}$, in the DoS-robust test diagnoser $G_{S C C}^{r o b}$, each automaton $G_{d, i}^{r o b}$, $i=1, \ldots, N$, is computed from a different automaton $G_{a, i}^{\ell}$.
} 
STEP 3. Verify if there exists a nontrivial SCC of $G_{S C C}^{r o b}$ formed with states $\left(x_{d}^{1}, x_{\ell}^{1}, x_{n}^{1}\right), \ldots,\left(x_{d}^{m}, x_{\ell}^{m}, x_{n}^{m}\right)$, such that, for all $p \in\{1, \ldots, m\}, x_{\ell}^{p}$ is an Y-labeled state, and $x_{d}^{p}=\left(x_{d}^{p, 1}, \ldots, x_{d}^{p, N}\right)$, where states $x_{d}^{p, i}, i=i, \ldots, N$, are uncertain states.

3.1. If the answer is Yes, then $L$ is not DoS-robustly codiagnosable with respect to $F_{a, i}, P_{a_{i}, s_{i}}: \Sigma_{a, i}^{*} \rightarrow \Sigma_{s, i}^{*}$, $i=1, \ldots, N$, and $\Sigma_{f}$; and so, return False. Otherwise, $L$ is DoS-robustly codiagnosable, and thus, return True.

Example 4. Let us revisit the NDES depicted in Figure 2, where $\Sigma_{o_{1,1}}=\{\beta\}, \Sigma_{o_{1,2}}=\{\alpha\}, \Sigma_{o_{2,2}}=\{\alpha\}, \Sigma_{o_{2} 3}=$ $\{\gamma, \delta\}, \Sigma_{u o}=\left\{\mu, \sigma_{f}\right\}$ and $\Sigma_{f}=\left\{\sigma_{f}\right\}$. As in Example 1, we assume that communication channel $c h_{2,2}$ is the unique channel that is vulnerable to DoS attacks.

In order to illustrate how Algorithm 2 works, consider sequence $s_{a}=\sigma_{f} t_{2,2} t_{2,2} t_{2,2_{d}} \alpha \alpha_{s_{1}} t_{2,2_{d}} \gamma \gamma_{s_{2}} \alpha_{s_{2}}\left(\delta \delta_{s_{2}}\right)^{p} \in$ $L\left(G_{S C C}^{r o b}\right), p \in \mathbb{Z}_{+}^{*}$. Let $P_{a, a_{i}}:\left(\Sigma_{a, 1} \cup \Sigma_{a, 2}\right)^{*} \rightarrow \Sigma_{a, i}^{*}, i=$ 1,2 . Then, we may obtain two sequences from $s_{a}$ : (i) $s_{a_{1}}=$ $P_{a, a_{1}}\left(s_{a}\right)=\sigma_{f} \alpha \alpha_{s_{1}} \gamma \delta^{p} \in L\left(G_{n, 1}\right)$, and (ii) $s_{a_{2}}=$ $P_{a, a_{2}}\left(s_{a}\right)=\sigma_{f} t_{2,2} t_{2,2} t_{2,2_{d}} \alpha t_{2,2_{d}} \gamma \gamma_{s_{2}} \alpha_{s_{2}}\left(\delta \delta_{s_{2}}\right)^{p} \in$ $L\left(G_{n, 2}\right)$. Notice that sequence $s_{a}$ models the case when the plant has generated the arbitrarily long failure sequence $\sigma_{f} \alpha \gamma \delta^{p}=P_{a_{1}}\left(s_{a_{1}}\right)=P_{a_{2}}\left(s_{a_{2}}\right)$, and local diagnosers $L D_{1}$ and $L D_{2}$ have observed sequences $P_{a_{1}, s_{1}}\left(s_{a_{1}}\right)=\alpha_{s_{1}}$ and $P_{a_{2}, s_{2}}\left(s_{a_{2}}\right)=\gamma_{s_{2}} \alpha_{s_{2}} \delta_{s_{2}}^{p}$, respectively. It can be seen, for normal sequences $w_{1}=\alpha \mu \delta^{p}$ and $w_{2}=\gamma \alpha \mu \delta^{p}$, that $\alpha_{s_{1}} \in P_{a_{1}, s_{1}}\left(F_{a, 1}\left(w_{1}\right)\right)$ and $\gamma_{s_{2}} \alpha_{s_{2}} \delta_{s_{2}}^{p} \in P_{a_{2}, s_{2}}\left(F_{a, 2}\left(w_{2}\right)\right)$. Therefore, it can be concluded that language $L(G)$ is not DoS-robustly codiagnosable with respect to $F_{a, i}, P_{a_{i}, s_{i}}$, $i=1,2$, and $\Sigma_{f}$. This is captured in Step 3 of Algorithm 2, since sequence $s_{a}$ reaches a state of $G_{S C C}^{r o b}$ that belongs to a nontrivial SCC formed with states $\left(x_{d}^{1}, x_{\ell}^{1}, x_{n}^{1}\right), \ldots$, $\left(x_{d}^{56}, x_{\ell}^{56}, x_{n}^{56}\right)$, where, for $p=1, \ldots, 56, x_{\ell}^{p}=x_{5} F$, and $x_{d}^{p}=\left(\left\{x_{3} F, x_{5} F, x_{7} N, x_{8} N\right\},\left\{x_{5} F, x_{8} N\right\}\right)$.

\section{CONCLUSION}

In this paper, a model for NDES subject to DoS attacks is introduced, so as to represent the adverse effects of DoS attacks on the observations of local diagnosers. Based on this new model, it is proposed a new property of robust codiagnosability that takes into account the possible effects of DoS attacks, referred here to as DoS-robust codiagnosability. It is also proposed a systematic way to verify whether a NDES subject to DoS attacks is DoSrobustly codiagnosable or not. We are currently working to apply the proposed model to address other problems, such as, supervisory control and opacity of NDES subject to DoS attacks.

\section{REFERENCES}

Alves, M.V.S., Carvalho, L.K., and Basilio, J.C. (2017). Supervisory control of timed networked discrete event systems. In 56th Conference on Decision and Control, 4859-4865. Melbourne, Australia.

Carvalho, L.K., Wu, Y., Kwong, R., and Lafortune, S. (2018). Detection and mitigation of classes of attacks in supervisory control systems. Automatica, 97, $121-$ 133.
Cassandras, C.G. and Lafortune, S. (2008). Introduction to Discrete Event Systems. Springer, New York, 2nd edition.

Debouk, R., Lafortune, S., and Teneketzis, D. (2000). Coordinated decentralized protocols for failure diagnosis of discrete event systems. Discrete Event Dynamic Systems, 10(1), 33-86.

Góes, R.M., Kang, E., Kwong, R., and Lafortune, S. (2017). Stealthy deception attacks for cyber-physical systems. In 56th Conference on Decision and Control, 4224-4230. Melbourne, Australia.

Kurose, J.F. and Ross, K.W. (2013). Computer Networking: A Top-Down Approach. Pearson, Upper Saddle River, NJ, USA, 6th edition.

Lima, P.M., Alves, M.V.S., Carvalho, L.K., and Moreira, M.V. (2019). Security against communication network attacks of cyber-physical systems. Journal of Control, Automation and Electrical Systems, 30(1), 125-135.

Lin, F. (2014). Control of networked discrete event systems: Dealing with communication delays and losses. SIAM Journal on Control and Optimization, 52(2), 1276-1298.

Lohachab, A. and Karambir, B. (2018). Critical analysis of DDoS-an emerging security threat over IoT networks. Journal of Communications and Information Networks, 3(3), 57-78.

Nunes, C.E.V., Moreira, M.V., Alves, M.V.S., Carvalho, L.K., and Basilio, J.C. (2018). Codiagnosability of networked discrete event systems subject to communication delays and intermittent loss of observation. Discrete Event Dynamic Systems, 28(2), 215-246.

Pasqualetti, F., Dörfler, F., and Bullo, F. (2013). Attack detection and identification in cyber-physical systems. IEEE Transactions on Automatic Control, 58(11), 27152729.

Ramadge, P.J.G. and Wonham, W.M. (1989). The control of discrete event systems. Proceedings of the IEEE, $77(1), 81-98$.

Sampath, M., Sengupta, R., Lafortune, S., Sinnamohideen, K., and Teneketzis, D. (1995). Diagnosability of discreteevent systems. IEEE Transactions on Automatic Control, 40(9), 1555-1575.

$\mathrm{Su}, \mathrm{R}$. (2018). Supervisor synthesis to thwart cyber attack with bounded sensor reading alterations. Automatica, $94,35-44$.

Viana, G.S., Alves, M.V.S., and Basilio, J.C. (2017). Codiagnosability of timed networked discrete-event systems subject to event communication delays and intermittent loss of observation. In 56th Conference on Decision and Control, 4211-4216. Melbourne, Australia.

Viana, G.S. and Basilio, J.C. (2019). Codiagnosability of discrete event systems revisited: A new necessary and sufficient condition and its applications. Automatica, 101, $354-364$.

Zargar, S.T., Joshi, J., and Tipper, D. (2013). A survey of defense mechanisms against distributed denial of service (DDoS) flooding attacks. IEEE Communications Surveys \& Tutorials, 15(4), 2046-2069. 